\title{
Ameliorative efficacy of Sepia officinalis ink extract on hepatorenal injury-induced following high-dose folic acid supplementation in rats
}

\author{
Mariam T Elrouby ${ }^{1}$, Sara B Ali ${ }^{2}$, Nagat Saleh Hossuin Moftha ${ }^{3}$, Abdeljalil Mohamed Al Shawoush ${ }^{2}$ and Ayman \\ S. Mohamed 2,*
}

${ }^{1}$ Faculty of biotechnology, October University for Modern Sciences and Arts, Egypt.

${ }^{2}$ Zoology Department - Faculty of Science - Cairo University, Egypt.

${ }^{3}$ Department of zoology, Faculty of arts and sciences Alabyar, University of Benghazi, Libya.

GSC Biological and Pharmaceutical Sciences, 2021, 17(03), 038-046

Publication history: Received on 30 October 2021; revised on 03 December 2021; accepted on 05 December 2021

Article DOI: https://doi.org/10.30574/gscbps.2021.17.3.0347

\begin{abstract}
Sepia ink, a black suspension of melanin granules, is a multifunctional marine bioactive material. The present study aims to evaluate the ameliorative effect of the ink extract (IE) of the cuttlefish (Sepia officinalis) during high dosage administration of the FA in rats. Kidney injury induced by a single oral dose of FA ( $250 \mathrm{mg} / \mathrm{kg}$ ). Eighteen male Wistar albino rats were the control, FA group, and FA+ IE group $(250 \mathrm{mg} / \mathrm{kg})$. The IE showed a significant ameliorative effect against hepatorenal injury induced by high intake of FA as evident by decreasing the levels of serum aminotransferases (AST and ALT), urea, creatinine, uric acid, and significantly increased total serum albumin. Treatment with IE normalized the antioxidant status of the injured animals by reducing the MDA and the significant increase in the levels of GSH and CAT. The present study revealed that IE had an insightful effect against hepatorenal injury-induced following high intake of FA in rats, as it alleviates the alterations in the oxidative stress markers.
\end{abstract}

Keywords: Sepia officinalis; Folic acid; Antioxidant; Ink; Oxidative stress

\section{Introduction}

Folate is a water-soluble vitamin that is required for cell growth and development [1]. The liver is the major organ for the storage and metabolism of folates [2]. Hypervitaminosis has been linked to liver injury, according to recent research [3]. In Egypt, folic acid (FA) is a synthetic folate that is added to fortify Baladi bread [4]. Increased folic acid use has been linked to liver damage (2015). According to new findings, folic acid is biotransformed into 5-methyl-tetrahydro folic acid (5-MTHF) in the hepatic portal vein [5]. Concerns about the safety of long-term high-dose folic acid supplementation that results in the accumulation of unmetabolized folic acid (UFA) have been growing [6]. Furthermore, Field et al. suggested that the development of liver injury as a result of UFA could be related to enzyme inhibition [7]. Unmetabolized homocysteine accumulates after a high dose of UFA, according to a significant body of research [6]. Unfortunately, evidence suggests that high levels of homocysteine are associated with negative consequences such as oxidative stress and non-alcoholic fatty liver disease (NAFLD) [8].

Acute kidney damage is a major public health problem that affects millions of people around the world [9]. Acute kidney injury (AKI) is defined by a sudden drop in renal function that is accompanied by multiple overlapping and complex pathophysiological processes [10]. Kidneys have become a powerful platform for a variety of duties, including maintaining general fluid balance, controlling and filtering minerals from the blood, filtering waste materials from food, drugs, and harmful chemicals, and so on. [11]. It has been discovered that one of the most important techniques for maintaining a normal folate level in the blood is efficient renal reabsorption. The rapid formation of folic acid crystals

\footnotetext{
* Corresponding author: Ayman S. Mohamed

Zoology Department - Faculty of Science - Cairo University, Egypt.
} 
in renal tubules, on the other hand, causes acute tubular necrosis and renal cortical scarring, resulting in acute renal failure (ARF) [12]. As a result, FA is one of the model chemicals commonly employed to research the pathophysiology of ARF [13]. Acute and chronic renal failure develops after FA treatment [14]. The development of ARF after a single dose of FA injection could be related to a general impairment in solute handling as a result of the suppression of the $\mathrm{Na}+/ \mathrm{K}+$ ATPase activity [15]. Furthermore, high-dose folic acid administration causes kidney damage, as evidenced by increased creatinine levels and tubule interstitial injury scores [15]. Acute renal failure (ARF) with high dosages of FA has been documented to be a severe clinical concern [16].

Unfortunately, traditional nephrotoxicity and hepatotoxicity medications are insufficient and can have substantial adverse effects. As a result, generating novel drugs from natural ingredients may reduce the risk of toxicity while maintaining therapeutic efficacy in clinical trials.

Chemicals produced by aquatic organisms can help us learn more about human ailments and how to cure them [17]. Undiscovered chemicals found in marine organisms lead to new molecular structures with varied features [18]. Many marine items (animal, algae, fungi, and bacteria) have recently been evaluated for bio-activities, such as antifungal and antibacterial properties [19].

Squid, octopus, and cuttlefish are cephalopod mollusks which have a unique defensive trait of spewing ink when assaulted. The cuttlefish Sepia officinalis ejects a black ink as it attacks [20]. This ink is a marine bioactive substance with several functions. It contains a variety of bioactive chemicals, including anticancer, antiviral, and antifungal agents [21]. Sepia ink extract also has anticancer activities [22], leukocyte-number-increasing characteristics, antioxidant characteristics [22], anti-retrovirus capabilities and antibacterial characteristics [23]. Sepia ink has previously been found to alleviate the in vivo immunosuppression caused by cyclophosphamide in mice [19]. The therapeutic effect of sepia ink extract against cholestasis-induced renal abnormalities in male rats was discovered by Soliman et al. [24]. The goal of this study was to see if the ink extract (IE) of the marine mollusk cuttlefish (Sepia officinalis) could protect rats against the effects of large doses of FA.

\section{Material and methods}

\subsection{Chemicals and reagents}

All drugs, chemicals, and solvents were purchased from local firms (Egypt), and they were of the highest purity and analytical grade.

\subsection{Preparation of cuttlefish ink extract (IE)}

Fresh cuttlefish (Sepia officinalis) were obtained from a fishmonger and transported quickly to the laboratory, where they were dissected and the ink collected. Using a lyophilizer, the extraction was reduced to a dark residue (LABCONCO lyophilizer, shell freeze system, USA).

\subsection{Animals, housing conditions, and diet}

The experimental animals used in this research were the adult male albino rats (Rattus norvegicus) weighing 150 - 170 g. The animals were obtained from National Research Center (NRC), Dokki, Cairo, Egypt. Animals were caged in groups of six and given food and water ad libitum. Rats were kept under fixed appropriate conditions of housing and handling. Rats were acclimatized to laboratory conditions for 7 days before commencement of the experiment.

\subsection{Experimental protocol}

The animals were divided into three groups, each with six animals. The first group (Control) consisted of rats that were given a single oral dose of $0.3 \mathrm{M} \mathrm{Na}_{2} \mathrm{CO}_{3}$ and then given dist. water every day for seven days. The animals in the second group (FA) received a single oral dosage of FA (250 mg/kg) [25] and then administered orally with dist. water daily for seven days. The third group (FA+ ink extract) received a single oral dosage of FA (250 mg/kg) before receiving 200 $\mathrm{mg} / \mathrm{kg}$ of ink extract orally for seven days [26].

\subsection{Animal Handling}

At the end of the experimental period, animals were euthanized by intraperitoneal injection sodium pentobarbital (50 $\mathrm{mg} / \mathrm{kg}$ body weight). Blood samples were obtained by cardiac puncture and then they were centrifuged at $3000 \mathrm{rpm}$ for 20 minutes. The clear non-hemolyzed sera were aspirated into sterilized Eppendorf tubes for each rat. The sera were kept in a deep freezer at $-80^{\circ} \mathrm{C}$ pending biochemical analysis. After blood collection, the rats were rapidly dissected. 
Pieces of liver and kidney from each rat were washed and fixed in neutral buffered formalin pending histological investigations.

\subsection{Tissue homogenate preparation}

Tissue were weighted and homogenized (10\% w/v) in ice-cold $0.1 \mathrm{M}$ Tris-HCl buffers (pH 7.4). The homogenate was centrifuged at $860 \times \mathrm{g}$ for $15 \mathrm{~min}$. and the resultant supernatant was saved in aliquots and stored at $-20^{\circ} \mathrm{C}$ for the biochemical analyses

\subsection{Histopathological Preparation}

Kidney and liver were excised and immersed in $10 \%$ formalin solution. Samples were embedded in paraffin. From the paraffin blocks, sections of $5 \mu \mathrm{m}$ in thickness were made and stained with hematoxylin and eosin to assess the pathological changes using light microscopy at 40X.

\subsection{Biochemical Assessment}

The appropriate kits (Biodiagnostic kits) were used for the determination of albumin level; serum aminotransferase enzymes (ASAT \& ALAT) activities, creatinine, urea, and uric acid were measured according to the manufacturer's instructions using Bio-diagnostic kits (Giza, Egypt). MDA level is a record of lipid peroxidation, glutathione reduced (GSH) and catalase were determined in the liver and kidney homogenates supernatant as indicated by the producer directions using Biodiagnostic (Giza, Egypt).

\subsection{Statistics}

All Values were expressed as means \pm standard error of mean (SEM). The comparisons within groups were evaluated utilizing one-way analysis of variance (ANOVA) with Duncan's post hoc test to compare the group means and $p<0.05$ was considered statistically significant. SPSS for Windows (version 15.0) was used for the statistical analysis.

\section{Results}

\subsection{Effects of Sepia officinalis ink extract (IE) on serum biomarkers}

The levels of the serum AST, ALT, albumin, creatinine, urea, and uric acid in control, untreated and IE-treated groups showed in Table 1 . The FA group showed a significant increase $(\mathrm{P}<0.05)$ in AST, ALT, creatinine, urea, and uric acid levels, while albumin decreased significantly compared to the control grou However, treatment with IE significantly decreased $(\mathrm{P}<0.05)$ the serum AST, ALT, creatinine, urea, and uric acid levels while albumin level increased compared to the FA grou

Table 1 Therapeutic effect of Sepia officinalis ink on kidney functions in folic acid treated rats

$\mid$\begin{tabular}{|l|c|c|c|}
\hline Parameters & Control & FA & FA+ IE \\
\hline AST $(\mathrm{U} / \mathrm{ml})$ & $6.80 \pm 1.20 \mathrm{a}$ & $13.23 \pm 0.49 \mathrm{c}$ & $10.1 \pm 0.49 \mathrm{~b}$ \\
\hline ALT $(\mathrm{U} / \mathrm{ml})$ & $138.1 \pm 2.05 \mathrm{a}$ & $153.01 \pm 1.69 \mathrm{c}$ & $142.82 \pm 1.76 \mathrm{~b}$ \\
\hline Albumin $(\mathrm{g} / \mathrm{dl})$ & $23.82 \pm 1.26 \mathrm{c}$ & $14.97 \pm 0.82 \mathrm{a}$ & $19.99 \pm 0.36 \mathrm{~b}$ \\
\hline Creatinine $(\mathrm{mg} / \mathrm{dl})$ & $0.31 \pm 0.03 \mathrm{a}$ & $1.27 \pm 0.04 \mathrm{c}$ & $0.57 \pm .05 \mathrm{~b}$ \\
\hline Urea $(\mathrm{mg} / \mathrm{dl})$ & $8.75 \pm .73 \mathrm{a}$ & $60.83 \pm 1.35 \mathrm{c}$ & $54.17 \pm 1.13 \mathrm{~b}$ \\
\hline Uric acid $(\mathrm{mg} / \mathrm{dl})$ & $3.98 \pm 0.05 \mathrm{a}$ & $5.51 \pm 0.13 \mathrm{c}$ & $4.65 \pm .05 \mathrm{~b}$ \\
\hline
\end{tabular}
Values are means \pm se (n = 6 per group). Each value not sharing a common letter superscript is significantly different $(\mathrm{P}<0.05)$.

\subsection{Effects of Sepia officinalis ink extract (IE) on oxidative stress markers}

Significant decreases $(\mathrm{p}<0.05)$ in GSH and CAT levels were observed in the FA-treated group compared to the control grou However, MDA concentration was increased significantly (Table 2). After the IE treatment, there has been a significant increase in GSH and CAT levels while MDA concentration decreased significantly compared to the FA grou 
Table 2 Therapeutic effect of Sepia officinalis ink on oxidative stress markers in folic acid treated rats

\begin{tabular}{|l|l|l|l|l|}
\hline Parameters & Tissue & Control & FA & FA+ IE \\
\hline \multirow{2}{*}{$\begin{array}{l}\text { MDA } \\
\text { (nmol/g.tissue) }\end{array}$} & Liver & $3.35 \pm 0.28^{\mathrm{a}}$ & $6.11 \pm 0.87^{\mathrm{c}}$ & $4.15 \pm 0.68^{\mathrm{b}}$ \\
\cline { 2 - 5 } & Kidney & $4.55 \pm 0.86^{\mathrm{a}}$ & $8.56 \pm 1.26^{\mathrm{c}}$ & $6.1 \pm 0.35^{\mathrm{b}}$ \\
\hline \multirow{2}{*}{$\begin{array}{l}\text { GSH } \\
(\mathrm{mg} / \text { g.tissue) }\end{array}$} & Liver & $17.57 \pm 2.38^{\mathrm{c}}$ & $8.93 \pm 1.34^{\mathrm{a}}$ & $17.57 \pm 2.38^{\mathrm{c}}$ \\
\cline { 2 - 5 } & Kidney & $19.7 \pm 0.49^{\mathrm{c}}$ & $9.6 \pm 0.42^{\mathrm{a}}$ & $14.23 \pm 0.60^{\mathrm{b}}$ \\
\hline \multirow{2}{*}{$\begin{array}{l}\text { CAT } \\
\text { (U/g.tissue) }\end{array}$} & Liver & $4.54 \pm 0.24^{\mathrm{c}}$ & $1.01 \pm 0.23^{\mathrm{a}}$ & $4.54 \pm 0.24^{\mathrm{c}}$ \\
\cline { 2 - 5 } & Kidney & $0.14 \pm 0.01^{\mathrm{c}}$ & $0.04 \pm 0.01^{\mathrm{a}}$ & $0.09 \pm 0.02^{\mathrm{b}}$ \\
\hline
\end{tabular}

Values are means \pm se $(\mathrm{n}=6$ per group). Each value not sharing a common letter superscript is significantly different $(\mathrm{P}<0.05)$.

\subsection{Histopathological analysis}

The liver of control rats is formed of the classic hepatic lobules. Blood sinusoids were seen separating the cords of the liver cells and lined by flattened endothelial cells and Von Kupffer cells (K) (Fig. 1). The histology of liver sections obtained from FA-treated rats showed the loss of hepatic lobular architecture morphological alterations, necrosis (N), and congested sinusoids (S) (Fig. 1). Liver sections of Ink-treated rats showed moderate to mild degenerated changes in hepatocytes and a clear improvement in the hepatic architecture (Fig. 1).

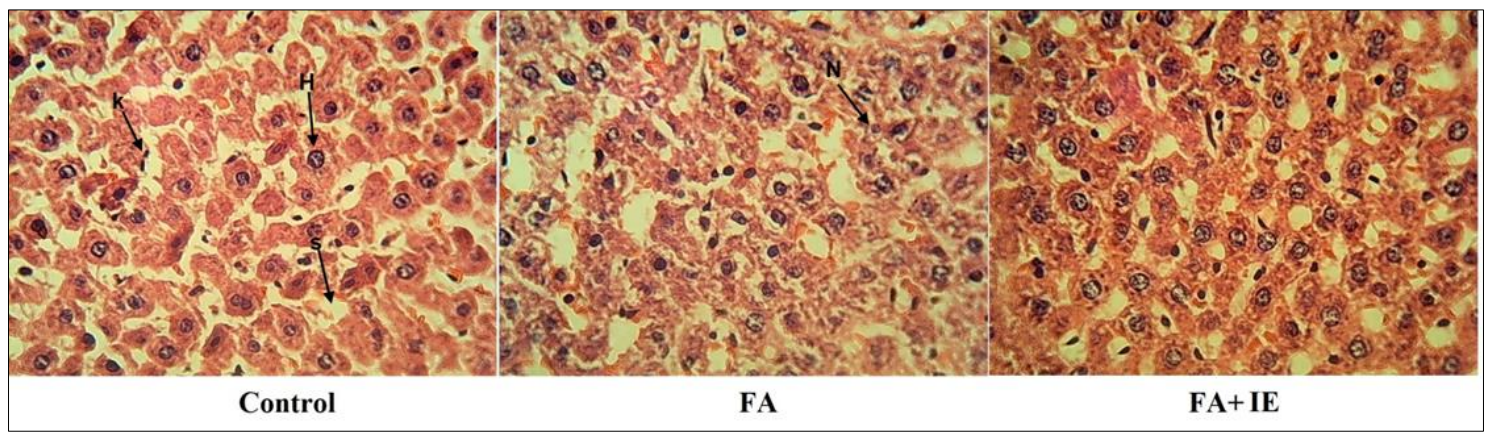

Figure 1 Histopathological examination of the liver. (H) Hebatocyte, (K) Von Kupffer cells, (N) necrosis, and (S) congested sinusoids

Microscopic examination of kidneys of control groups showed normal histoarchitecture of the tissue. Glomeruli (G) appear as dense tufts of capillaries enclosed in the outer layer of Bowman capsules. Numerous renal tubules (r) were observed (Fig. 2). On the other hand, FA-treated rats showed severe degeneration in the glomerulus with deformed renal tissue architecture (Fig. 2). In contrast, the treatment with ink caused marked regeneration in the glomerulus and Bowman's capsule with typical renal tissue architecture (Fig. 2).

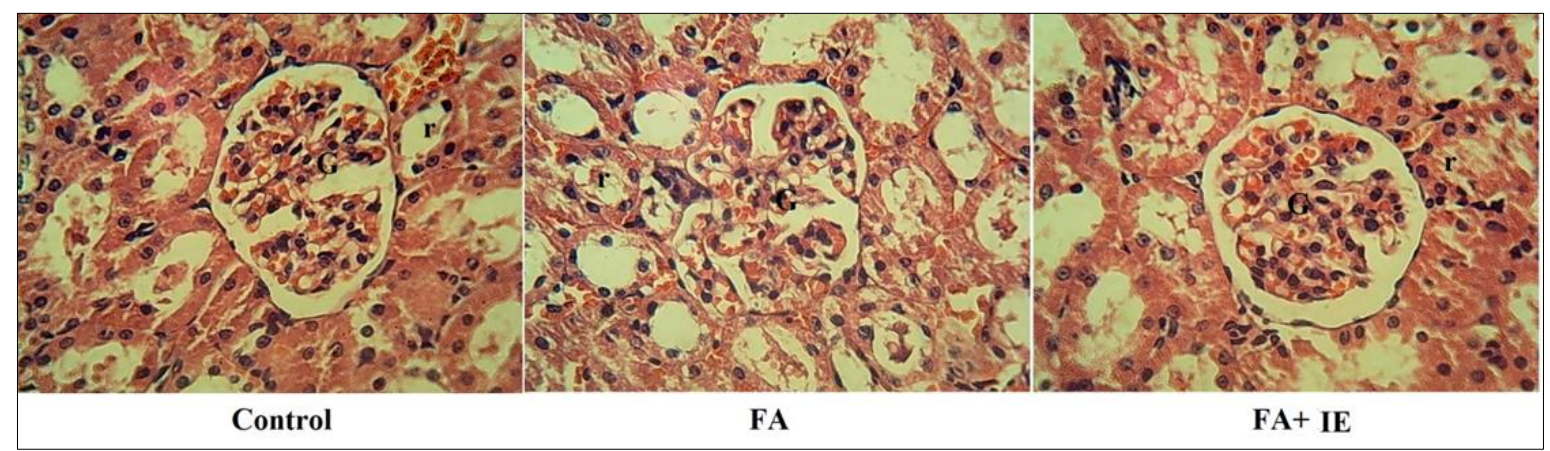

Figure 2 Histopathological examination of the kidney. (G) Glomeruli and (r) renal tubules 


\section{Discussion}

MAFLD (metabolic dysfunction-associated fatty liver disease) is a leading cause of morbidity and mortality, as well as a major public health issue globally [27]. The hunt for selective and less toxic molecules for the treatment of liver disease is ongoing. Natural chemicals have a low toxicity and show very few side effects when used as a protective liver remedy. In marine species, one of the most important suppliers of these natural chemicals. Endogenous synthesis of protective compounds has been shown as a selection advantage for many marine invertebrates [28]. Given the link between oxidative stress and chronic liver disease, it's been hypothesised that consuming antioxidants can help prevent fibrosis by triggering the stress response [29]. The beneficial effects of melanin on liver structure and function have been proven [30]. The liver impairment generated by high dosages of FA in this investigation resulted in hyperhomocysteinemia, which interfered with melanin biosynthesis. Assume, then, that the novel hepatic protective medication can preserve liver stability during large doses of FA by acting as both an antioxidant and a melanin reservoir. It could be a promising liver protective medication in that circumstances. As a result, the current investigation shed information on the Sepia officinalis ink extract (IE)'s protective role during high-dose administration of the FA in rats.

Biochemical marker enzymes, such as serum aminotransferases, AST, and ALT, are commonly used to measure liver damage and are used for accurate detection and early diagnosis. The liver plays a crucial role in folate metabolism [31]. The increased activity of the serum enzymes ASAT and ALAT in the current investigation could be attributed to a strong link with the hyperhomocysteinemia condition that occurs after high FA administration [32]. The findings of this investigation backed up those of Obeid and Herrmann, who found that homocysteine altered intracellular lipid metabolism and promoted hepatic fat storage [33]. Increased activity of the enzymes investigated as a result of disruption in their biosynthesis, as well as changes in the permeability of the liver membrane. In high FA-treated rats, treatment with IE extract dramatically reduced serum AST and ALT activity, showing that the hepatic cell membrane's functional integrity was maintained.

Albumins are anti-inflammatory proteins that indicate a problem with synthetic liver function. The present investigation found a considerable decrease in albumin content following high FA treatment in rats, similar to the findings of Chilom et al. [34]. The drop in albumin level could be due to hepatic cell malfunction, resulting in the liver's ability to synthesis albumin being reduced. Furthermore, the decrease in albumin levels could be owing to its role as an FA transporter in the blood [34]. Treatment with IE, on the other hand, restores albumin levels to near-normal levels. Albumin levels returning to normal indicate hebetic cell regeneration. In addition, the histopathological finding in the present investigation confirmed the regeneration of the hepatic cells that affirmed their ability to re-synthesis the albumin.

Nephrotoxicity is a kidney disorder caused by exposure to a variety of harmful xenobiotics, such as medications and environmental pollutants. Furthermore, the prevalence of acute renal failure (ARF) and chronic kidney disease (CKD) has steadily increased in recent years [9]. There has been a significant increase in the consumption of folic acid (FA), which is used to treat anaemia and vitamin deficiencies. Furthermore, there is a strong link between FA overdoses and kidney damage [35]. Finding a safe and antioxidant chemical that can block renal damage is critical for the prevention of renal injury and disease during FA treatment. As a result, the goal of this investigation was to see if the antioxidant sepia ink extract (IE) could protect against renal impairment caused by FA.

The loss of kidney function that occurs as a result of renal disease ranges from an increase in serum creatinine to renal failure. Indeed, the most extensively utilized markers for nephrotoxicity and kidney injury have historically been serum urea and creatinine concentrations. The results of the present study showed a significant increase in the serum urea, uric acid, and creatinine following FA administration. The rise in serum kidney function indices after FA administration could be attributable to renal injury, such as a decrease in creatinine clearance due to an overall impairment in solute handling caused by inhibition of the $\mathrm{Na}+\mathrm{K}+\mathrm{ATPase}$ activity [15]. [36] confirmed this interpretation, stating that high dose FA treatment resulted in a decrease in creatinine clearance, which can occur despite an increase in renal blood flow. Furthermore, in the current study, the results of the histological analysis of kidney tissues revealed glomerular atrophy and enlargement of the proximal tubules, which could be attributable to secondary brush border destruction or a decrease in the height of the lining cells as a result of degeneration. Furthermore, higher net tubular absorption may result in hyperuricemia once a large dose of FA is administered.

On the other hand, oral co-administration of IE with FA resulted in a considerable reduction in serum urea, uric acid, and creatinine concentrations. According to the current findings, IE has a strong protective impact, which is supported by its ability to restore the analyzed renal function parameters to near-control levels, which could be owing to a contributing self-healing mechanism that restores kidney structure and function. The current study backed with the 
findings of Hung et al., who found that melanin, one of the most prevalent components of the IE, possesses renoprotective properties [37].

According to a study, there is a substantial correlation between the accumulation of unmetabolized homocysteine and oxidative stress after consuming a high quantity of UFA. [32]. Due to lipid peroxidation, MDA is one of the highly reactive metabolic products produced [38]. Increased MDA levels have been linked to increased lipid peroxidation [2]. MDA increased after high dosages of FA addition, which appears to validate the current findings [8]. It has been shown that homocysteine may easily self-oxidize to produce ROS and can activate NADPH oxidase to produce ROS [39] and can activate NADPH oxidase to generate ROS [40].

MDA levels, on the other hand, were considerably lower in rats given IE. Reduced lipid peroxidation-mediated oxidative stress, on the other hand, could be a viable and successful method for avoiding and treating hepatorenal damage. The findings of Fahm et al., who reported that IE greatly reduced MDA levels in injured liver and kidney tissues, implying that IE's injury-protective mechanism is due to its antioxidant action [18]. Reduced glutathione (GSH) is a critical component of the intracellular protective mechanisms that scavenge free radicals in all cell types [41]. GSH protects against free radicals and reactive oxygen intermediates by neutralising them. Indeed, the SH group of cysteine, an amino acid component of GSH, has a strong electron-donating ability, neutralising the hydroxyl radical and plays an important role against inflammation and oxidative stress [42]. Following high dose administration of FA, there may be a shortage of non-enzymatic antioxidant GSH as a result of increased use for trapping free radicals. In comparison to controls, the level of GSH in the hepatorenal tissues was significantly reduced in the current study's results. GSH levels are restored after treatment with IE, which may be attributable to its antioxidant action.

Catalase (CAT) is an essential enzyme that catalyzes the elimination of hydrogen peroxide produced by the SODcatalyzed process. In line with the findings of Stallons et al., the current investigation found that high-dose FA treatment resulted in a decrease in CAT activity in hepatorenal tissues, which could be due to mitochondrial toxicity-induced AKI [43]. The increased homocysteine (Hcy)-mediated superoxide generation from NADPH oxidase, which is a potent inhibitor of catalase, implies that the FA group's catalase function is inhibited. However, after treatment with IE, renal CAT activity increased considerably in FA rats, which could be owing to its antioxidant impact and ability to scavenge free radicals.

\section{Conclusion}

The current study demonstrates that IE increased antioxidant state and avoided hepatorenal damage under high FA dosages. Furthermore, IE demonstrated a strong protective impact, as evidenced by its capacity to restore the examined liver and renal function parameters to near-control levels, which could be owing to a contributing self-healing mechanism that restores tissue structure and function. Finally, our research shown that IE can be used as a supplemental protective medication during hepatorenal damage.

\section{Compliance with ethical standards}

\section{Acknowledgments}

This work was supported by Cairo University.

\section{Disclosure of conflict of interest}

The authors have no conflicts of interest to declare that are relevant to the content of this article.

\section{Declarations of interest}

All Authors decelerate that no conflict of interest.

Ayman S. Mohamed decelerate that no conflict of interest.

\section{Statement of ethical approval}

The experiments were carried out according to international guidelines for care and use of laboratory animals. The protocol was approved by the Faculty of Science, Cairo University, Egypt, and Institutional Animal Care and Use Committee (IACUC), (Egypt). 


\section{References}

[1] Ducker GS, Rabinowitz JD. One-carbon metabolism in health and disease. Cell Metabol. 2017; 25: 27-42.

[2] Sid V, Siow YL, Karmin O. Role of folate in nonalcoholic fatty liver disease. Canadian Journal of Physiology and Pharmacology. 2017; 95(10).

[3] Alexander M, Kumar SR, Steinberg J, Garcia-Buitrago MT, Arosemena B, Leopoldo R. Liver Damage due to Hypervitaminosis. ACG Case Rep J. 2020; 7(7): e00431.

[4] Elhakim N, Laillou A, El Nakeeb A, Yacoub R, Yacoub M. Fortifying baladi Bread in Egypt: Reaching More than 50 Million People through the Subsidy Program. Food and Nutrition Bulletin. 2008; 33(4): S260-S271.

[5] Wright AJA, Dainty JR, Finglas PM. Folic acid metabolism in humansubjects revisited: potential implications for proposed mandatory folic acid fortification in the UK. Br J Nutr. 2007; 98: 667-675.

[6] Koseki K, Maekawa Y, Bito T. High-dose folic acid supplementation results in significant accumulation of unmetabolized homocysteine, leading to severe oxidative stress in Caenorhabditis elegans. Redox Biol. 2020; 37: 101724 .

[7] Field MS, Szebenyi DM, Stover PJ. Regulation of de novo purine biosynthesis by methenyltetrahydrofolate synthetase in neuroblastoma. J Biol Chem. 2006; 281: 4215-4221.

[8] Dai H, Wang W, Tang X, Chen R, Chen Z, Lu Y, et al. Association between homocysteine and non-alcoholic fatty liver disease in Chinese adults: a cross-sectional study. Nutr. J. 2016; 15: 102.

[9] Andreucci M, Faga T, Pisani A, Perticone M, Michael A. The ischemic/nephrotoxic acute kidney injury and the use of renal biomarkers in clinical practice. Eur J Intern Med. 2017; 39: 1-8.

[10] Joana Gameiro *JAFCOaJAL. Acute Kidney Injury: From Diagnosis to Prevention and Treatment Strategies. J Clin Med. 2020; 9(6): 1704.

[11] Shelton M, Park B, Copple IM. Role of Nrf2 in protection against acute kidney injury. Kidney Int. 2013; 84(6): 1090-1095.

[12] Stallons LJ WRSR. Suppressed mitochondrial biogenesis in folic acid-induced acute kidney injury and early fibrosis. Toxicol Lett. 2014; 224(3): 326-332.

[13] Xu , Qin , Li , Sun , Wang J, Liang, et al. Efficacy of Folic Acid Therapy on the Progression of Chronic Kidney Disease: The Renal Substudy of the China Stroke Primary Prevention Trial. JAMA Internal Medicine. 2016; 176(10): 1443-1450.

[14] Ortega A, Ramila D, Izquierdo A, Gonzalez L, Barat A, Gazapo R, et al. Role of the renin-angiotensin system on the parathyroid hormone-related protein overexpression induced by nephrotoxic acute renal failure in the rat. J Am Soc Nephrol. 2005; 16(4): 939-949.

[15] Jiang K, Ponzo TA, Tang H, Mishra PK, Macura SI, Lerman LO. Multiparametric MRI detects longitudinal evolution of folic acid-induced nephropathy in mice. Am. J. Physiol. Ren. Physiol. 2018; 315: F1252-F1260.

[16] Aparicio-Trejo OE, Reyes-Fermin LM, Briones-Herrera A, Tapia E, Leon-Contreras JC. Protective effects of Nacetyl-cysteine in mitochondria bioenergetics, oxidative stress, dynamics and S-glutathionylation alterations in acute kidney damage induced by folic acid. Free Radic Biol Med. 2019; 130: 379-396.

[17] Koyama T, Chounan R, Uemura D, Yamaguchi Y, Yazawa K. Hepatoprotective Effect of a Hot-Water Extract from the Edible Thorny Oyster Spondylus varius on Carbon Tetrachloride-Induced Liver Injury in Mice. Biosci Biotechnol Biochem. 2006; 70(3): 729-731.

[18] Fahmy SR, Soliman AM, Ali EM. Antifungal and antihepatotoxic effects of sepia ink extract against oxidative stress as a risk factor of invasive pulmonary aspergillosis in neutropenic mice. Afr J Tradit Complement Altern Med. 2014; 11(3): 148-159. 
[19] Neifar A, Rebeh FB, Gargouri A, Abdelmouleh A. Physicochemical characterisation of Sepia officinalis ink and the effects of storage conditions on the coagulation process. J. Mar. Biol. Assoc. UK. 2009; 89(4): 803-807.

[20] Palumbo A. Melanogenesis in the ink gland of Sepia officinalis. Pigment Cell Res. 2003; 16(5): 517-522.

[21] Liu H, Luo P, Chen S, Shang J. Effects of Squid Ink on Growth Performance, Antioxidant Functions and Immunity in Growing Broiler Chickens. Asian-Australian J. Anim. Sci. 2011; 24(12): 1752-1756.

[22] Soliman M, Fahmy. In vitro antioxidant, analgesic and cytotoxic activities of Sepia officinalis ink and Coelatura aegyptiaca extracts. Afr. J. Pharm. Pharmacol. 2013; 7(22): 1512-1522.

[23] Xie G, He S. Study of sepia improving natural killer cell activity in mice. J. Chin. Med. Univ. 2002; 1: 23-24.

[24] Soliman AM, Marie MAS, Saleh HM, Mohamed AS. Assessment of sepia ink extract role against. The Journal of Basic \& Applied Zoology. 2014; 67: 173-181.

[25] Gupta A, Puri V, Sharma R, Puri S. Folic acid induces acute renal failure (ARF) by enhancing renal prooxidant state. Exp Toxicol Pathol. 2012; 64(3): 225-232.

[26] Saleh H, Soliman AM, Mohamed AS, Marie MAS. Antioxidant effect of sepia ink extract on extrahepatic. Biomed Environ Sci. 2015; 28(8): 582-594.

[27] Eslam M, Sanyal AJ, George J. Consensus-Driven Proposed Nomenclature for Metabolic Associated Fatty Liver Disease. , International Consensus Panel. 2020; 158(7): 1999-2014.e1.

[28] Caldwell RL. An observation of inking behavior protecting adult Octopus bocki from predation by Green Turtle (Chelonia mydas) hatchlings. Pac. Sci. 2005; 59(1): 69-72.

[29] Uchida D, Takaki A, Oyama A, Adachi T, Wada N, Onishi H, et al. Oxidative Stress Management in Chronic Liver Diseases and Hepatocellular Carcinoma. Nutrients. 2020; 12(6): 1576.

[30] Belemets N, Kobyliak N, Virchenko O, Falalyeyeva T. Effects of polyphenol compounds melanin on NAFLD/NASH prevention. Biomedicine and Pharmacotherapy. 2017; 88: 267-276.

[31] Bailey LB, Caudill MA. Present knowledge in nutrition. 10th ed. Erdman JW, editor. Oxford (UK): WileyBlackwell. 2012.

[32] Koseki K, Maekawa Y, Bito T, Yabuta Y, Watanabe F. High-dose folic acid supplementation results in significant accumulation of unmetabolized homocysteine, leading to severe oxidative stress in Caenorhabditis elegans. Redox Biol. 2020; 37: 101724.

[33] Obeid R, Herrmann W. Homocysteine and lipids: S-adenosyl methionine as a key intermediate. FEBS Lett. 2009; 385: 1215-25.

[34] Chilom CG, Bacalum M, Stanescu MM, Florescu M. Insight into the interaction of human serum albumin with folic acid: A biophysical study. Spectrochimica Acta Part A: Molecular and Biomolecular Spectroscopy. 2018; 204: 648-656.

[35] Kumar D, Singla S, Puri V, Puri S. The restrained expression of NF-kB in renal tissue ameliorates folic acid induced acute kidney injury in mice. PLoS One. 2015; 10(1): e115947.

[36] Ortiz A, Husi H, Gonzalez-Lafuente L, Valino-Rivas L, Fresno M, Sanz AB, et al. Mitogen-Activated Protein Kinase 14 Promotes AKI. J. Am. Soc. Nephrol. JASN. 2017; 28: 823-836.

[37] Hung YC, Huang GS, Lin LW, Hong MY, Se PS. Thea sinensis melanin prevents cisplatin-induced nephrotoxicity in mice. Food Chem Toxicol. 2007; 45(7): 1123-30.

[38] Kaçmaz A, Polat A, User Y, Tilki M, Ozkan S, Sener G. Octreotide improves reperfusion-induced oxidative injury in acute abdominal hypertension in rats. J. Gastrointest. Surg. 2004; 8: 113-119.

[39] Olszewski AJ, McCully KS. Homocysteine metabolism and the oxidative modification of proteins and lipids. Free Radic. Biol. Med. 1993; 14: p 683-693. 
[40] Yaw LS, Kathy KWA, Connie WHW, Karmin O. Homocysteine stimulates phosphorylation of NADPH oxidase p47phox and p67phox subunits in monocytes via protein kinase C $\beta$ activation. Biochem. J.. 2006; 398: p 73-82.

[41] Pener G KLYMGNAÝ. Hepatic fibrosis in biliary-obstructed rats is prevented by Ginkgo biloba treatment. World J Gastroenterol. 2005; 11(35): 444-9.

[42] Circu ML,ATY. Redox biology of the intestine. Free Radic. Res. 2011; 45: 1245-1266.

[43] Stallons LJ, Whitaker RM, Schnellmann RG. Suppressed Mitochondrial Biogenesis in Folic Acid-Induced Acute Kidney Injury and Early Fibrosis. Toxicol Lett. 2014; 224(3): 326-332. 